\title{
Magnetoconductance through a chain of rings with or without periodically modulated spin-orbit interaction strength and magnetic field
}

\author{
B. Molnár, ${ }^{1}$ P. Vasilopoulos, ${ }^{2}$ and F. M. Peeters ${ }^{1}$ \\ ${ }^{1}$ Departement Fysica, Universiteit Antwerpen (Campus Drie Eiken), B-2610 Antwerpen, Belgium \\ ${ }^{2}$ Department of Physics, Concordia University, 1455 de Maisonneuve Quest, Montréal, Quebec, Canada H3G 1 M8
}

(Received 22 July 2004; revised manuscript received 19 May 2005; published 12 August 2005)

\begin{abstract}
Ballistic electron transport through a chain of quantum circular rings is studied in the presence of spin-orbit interaction (SOI) and of a perpendicular magnetic field $B$. From the analytic expressions for the transmission and reflection coefficients for a single ring we obtain the conductance through a chain of rings as a function of the SOI strength $\alpha$, the field $B$, and of the wave vector $k$ of the incident electron. Due to destructive spin interferences caused by the SOI the chain can be totally opaque for certain ranges of $k$ the width of which depends on the values of $\alpha$ and $B$. Outside these ranges the conductance oscillates with maximum values between $e^{2} / h$ and $2 e^{2} / h$. The effect of a periodic modulation of $\alpha$ and/or $B$ on the magnetoconductance is also investigated. A periodic, square-wave magnetoconductance pattern results within wide stripes in the parameter space spanned by $k, \alpha$, and $B$. Finite temperatures smoothen the square-wave profile of the conductance but do not alter its periodic character.
\end{abstract}

DOI: $10.1103 /$ PhysRevB.72.075330

PACS number(s): 72.25.-b, 71.70.Ej, 03.65.Vf, 85.35.-p

\section{INTRODUCTION}

In recent years the study of spintronics devices, which utilize the spin rather than the charge of an electron, has been intensified mainly because they are expected to operate at much higher speeds than the conventional ones and have potential applications in quantum computing. One such device is a single ring in the presence of the Rashba coupling ${ }^{1}$ or spin-orbit interaction (SOI) which results from asymmetric confinement in semiconductor nanostructures. It is important in materials with a small band gap such as InGaAs. An important feature of electron transport through a ring is that, even in the absence of an external magnetic field, the difference in the Aharonov-Casher phases of carriers traveling clockwise and counterclockwise produces spin-sensitive interference effects. ${ }^{2,3}$

In previous work we studied the influence of SOI on electron transport in the presence of a perpendicular magnetic field ${ }^{3} B$ and briefly on transport through a chain of rings in the absence of the field ${ }^{4} B$. Here we study in detail transport through a chain of identical rings in the presence of SOI of strength $\alpha$ and of a magnetic field $B$. In addition, we study the influence of periodic modulations of $\alpha$ or $B$ on the magnetoconductance. ${ }^{3,4}$

In Sec. II we obtain the transfer matrix for a single ring analytically and calculate the spin-dependent conductance as a function of the strength $\alpha$, the magnetic field $B$, and of the incident wave number $k$. Using these results we formulate the multiring transport in Sec. III. In Sec. IV we present numerical results and in Sec. V concluding remarks.

\section{ONE-RING TRANSPORT}

\section{A. Hamiltonian}

In the presence of SOI and of a magnetic field $B$ perpendicular to the $x-y$ plane of the ring the appropriate Hamiltonian $^{5}$ can be cast in the form ${ }^{3}$

$$
H=\hbar \Omega\left[\left(-i \frac{\partial}{\partial \varphi}-\frac{\Phi_{A B}}{2 \pi}+\frac{\omega_{s o}}{2 \Omega} \sigma_{r}(\varphi)\right)^{2}-\frac{\omega_{s o}^{2}}{4 \Omega^{2}}\right],
$$

where the operator $\sigma_{r}(\varphi)$ is given in terms of the Pauli matrices $\sigma_{x}, \sigma_{y}$, as $\sigma_{r}(\varphi)=\cos \varphi \sigma_{x}+\sin \varphi \sigma_{y}$. In this Hamiltonian the magnetic field $B$ appears through the AharonovBohm flux $\Phi_{A B}=\pi e a^{2} B / \hbar$ with $a$ the ring radius. The Zeemann term is neglected because it is very small for the ranges of the parameters we use, cf. Sec. IV. Further, $\Omega$ $=\hbar / 2 m^{*} a^{2}$ and $\omega_{s o}=\alpha / \hbar a$ is the frequency associated with the SOI. The parameter $\alpha$ represents the average electric field along the $z$ direction. For an InGaAs-based system the Rashba SOI strength $\alpha$ can be controlled by a gate voltage ${ }^{6}$ with values in the range $(0.5-2.0) \times 10^{-11} \mathrm{eV} \mathrm{m}$.

The eigenvalues, in units of $\hbar \Omega$, and the unnormalized eigenstates are given, respectively, by

$$
E_{n}^{(\mu)}=\left[n-\left(\Phi_{A B}+\Phi_{A C}^{(\mu)}\right) / 2 \pi\right]^{2}-\omega_{s o}^{2} / 4 \Omega^{2}
$$

and

$$
\Psi_{n}^{(\mu)}(\varphi)=e^{i n \varphi} \chi^{(\mu)}(\varphi), \quad \mu=1,2 .
$$

The spin-dependent term $\Phi_{A C}^{(\mu)}$ is the Aharonov-Casher phase

$$
\Phi_{A C}^{(\mu)}=-\pi\left[1+(-1)^{\mu}\left(\omega_{s o}^{2}+\Omega^{2}\right)^{1 / 2} / \Omega\right] .
$$

In terms of the eigenvectors $\left(\begin{array}{l}1 \\ 0\end{array}\right),\left(\begin{array}{l}0 \\ 1\end{array}\right)$ of the Pauli matrix $\sigma_{z}$ the orthogonal spinors $\chi^{(\mu)}(\varphi)$ are written as

$$
\chi^{(1)}(\varphi)=\left(\begin{array}{c}
\cos \frac{\theta}{2} \\
e^{i \varphi} \sin \frac{\theta}{2}
\end{array}\right), \quad \chi^{(2)}(\varphi)=\left(\begin{array}{c}
\sin \frac{\theta}{2} \\
-e^{i \varphi} \cos \frac{\theta}{2}
\end{array}\right),
$$

with the angle $\theta$ given by

$$
\theta=-\arctan \left(\omega_{s o} / \Omega\right) .
$$


Using the time-dependent Schrödinger equation with the Hamiltonian (1) one can show that the following continuity equation is valid for an arbitrary spinor wave function $\Psi(\varphi, t)=\left(\begin{array}{l}\Psi_{1}(\varphi, t) \\ \Psi_{2}(\varphi, t)\end{array}\right)$ :

$$
\frac{\partial \rho}{\partial t}+\frac{\partial J}{\partial \varphi}=0,
$$

where $\rho=\Psi^{\dagger} \Psi=\Psi_{1}^{*} \Psi_{1}+\Psi_{2}^{*} \Psi_{2}$ is the probability density. The probability current density $J$ is given by

$$
J=2 \operatorname{Re}\left\{\Psi^{\dagger}\left(-i \frac{\partial}{\partial \varphi}-\frac{\Phi_{A B}}{2 \pi}+\frac{\omega_{s o}}{2 \Omega} \sigma_{r}\right) \Psi\right\} .
$$

An arbitrary wave function can be expanded in terms of $\Psi(\varphi, t)=\Sigma_{\mu=1,2} \phi^{(\mu)}(\varphi, t) \chi^{(\mu)}(\varphi)$. Then the corresponding charge density is the sum of the spin-probability densities $\rho^{(\mu)}=\phi^{(\mu)}\left(\phi^{(\mu)}\right)^{*}(\mu=1,2): \rho=\rho^{(1)}+\rho^{(2)}$. As for the electric charge current, it also can be decomposed as $J=J^{(1)}+J^{(2)}$ with

$$
J^{(\mu)}=2 \operatorname{Re}\left\{\left(\phi^{(\mu)}\right)^{*}\left(-i \frac{\partial}{\partial \varphi}-\frac{\Phi_{A B}}{2 \pi}-\frac{\Phi_{A C}^{(\mu)}}{2 \pi}\right) \phi^{(\mu)}\right\},
$$

where $J^{(\mu)}$ is the spin-current density corresponding to spin polarization $\mu$. It can be also shown that the continuity equation holds for each spin component $\mu$, i.e.,

$$
\frac{\partial \rho^{(\mu)}}{\partial t}+\frac{\partial J^{(\mu)}}{\partial \varphi}=0 .
$$

\section{B. Boundary conditions, transfer matrix}

The ring connected to two leads is shown in Fig. 1. The appropriate boundary conditions are a spin-dependent version of Griffith's boundary conditions. ${ }^{7,8}$ In fact, at each junction the wave function must be continuous and the sum of the spin probability currents $J^{(\mu)}$ coming from the different parts of the device must vanish.

Using the coordinate representation the wave functions in the leads I and II can be expanded as

$$
\left\langle x \mid \Psi_{I}\right\rangle=\sum_{\mu=1,2}\left(c_{1}^{\mu} e^{-i k x}+c_{2}^{\mu} e^{i k x}\right) \chi^{(\mu)}(\pi),
$$

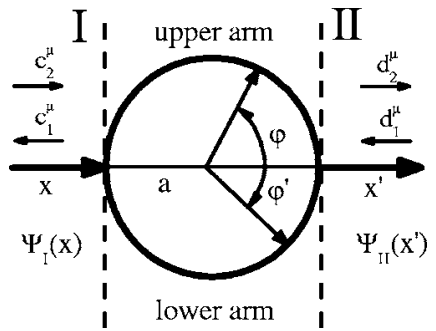

FIG. 1. Schematic of a single ring with the attached leads (regions I and II).

$$
\left\langle x^{\prime} \mid \Psi_{I I}\right\rangle=\sum_{\mu=1,2}\left(d_{1}^{\mu} e^{-i k x^{\prime}}+d_{2}^{\mu} e^{i k x^{\prime}}\right) \chi^{(\mu)}(0),
$$

with $k$ denoting the incident wave number. The coefficients $c_{2}^{\mu}\left(d_{1}^{\mu}\right)$ are the amplitudes of the spin state $(\mu=1,2)$ for electrons incident from the left (right) while $c_{1}^{\mu}\left(d_{2}^{\mu}\right)$ are those of the spin state exiting from the left (right) of the ring.

A similar expansion can be made in the upper $(u)$ and lower $(l)$ arm of the ring:

$$
\begin{gathered}
\left\langle\varphi \mid \Psi_{u}\right\rangle=\sum_{\mu, j=1,2} a_{j}^{\mu} e^{i n_{j}^{\mu} \varphi} \chi^{\mu}(\varphi), \\
\left\langle\varphi^{\prime} \mid \Psi_{l}\right\rangle=\sum_{\mu, j=1,2} b_{j}^{\mu} e^{-i n_{j}^{\mu} \varphi^{\prime}} \chi^{\mu}\left(-\varphi^{\prime}\right),
\end{gathered}
$$

where

$$
n_{j}^{\mu}=(-1)^{j}\left[k^{2} a^{2}+\left(\omega_{s o} / 2 \Omega\right)^{2}\right]^{1 / 2}+\Phi_{A B}+\Phi_{A C} .
$$

Equation (13) ensures the conservation of energy $E_{n^{\mu}}^{\mu}=k^{2} a^{2}$ at the junction points.

Applying the boundary conditions one can verify that the coefficients $d_{i}^{\mu}$ in lead II are connected to those $\left(c_{i}^{\mu}\right)$ in lead $\mathrm{I}$ in the following manner:

$$
\begin{gathered}
d_{1}^{\mu}=\left(1 / t^{\mu}\right) c_{1}^{\mu}-\left(r^{\mu} / t^{\mu}\right) c_{2}^{\mu}, \\
d_{2}^{\mu}=\left(r^{\mu} / t^{\mu}\right) c_{1}^{\mu}-\left(t^{\mu}-r^{\mu} / t^{\mu}\right) c_{2}^{\mu} .
\end{gathered}
$$

Here the complex coefficients $t^{\mu}$ and $r^{\mu}$ are given by

$$
\begin{aligned}
t^{\mu} & =\frac{8 i k q \cos \left(\Phi_{A B} / 2+\Phi_{A C}^{\mu} / 2\right) \sin (q a \pi)}{[1-\cos (2 q a \pi)] k^{2}+4 q^{2}\left[\cos \left(\Phi_{A B}+\Phi_{A C}^{\mu}\right)-\cos (2 q a \pi)\right]+4 i k q \sin (2 q a \pi)}, \\
r^{\mu} & =\frac{[1-\cos (2 q a \pi)] k^{2}+4 q^{2}\left[\cos (2 q a \pi)-\cos \left(\Phi_{A B}+\Phi_{A C}^{\mu}\right)\right]}{[1-\cos (2 q a \pi)] k^{2}+4 q^{2}\left[\cos \left(\Phi_{A B}+\Phi_{A C}^{\mu}\right)-\cos (2 q a \pi)\right]+4 i k q \sin (2 q a \pi)},
\end{aligned}
$$

where $q=\left[k^{2}+\left(\omega_{s o} / 2 a \Omega\right)^{2}\right]^{1 / 2}$. We would like to point out that in Ref. 3 we neglected the term $\hbar \omega_{s o}^{2} / 4 \Omega$ in the Hamiltonian given by Eq. (1) since for the situation considered there it was negligible compared to the values of $k a$ used. Here we must retain it in view of the periodic modulations of $\alpha$ and the wider range of $k a$ values considered. The formulas 
for $r^{\mu}$ and $t^{\mu}$ in Ref. 3 can be obtained from Eqs. (15) by replacing in them $q$ by $k$.

Instead of using the coordinate representation let us introduce the following notations in lead I:

$$
\begin{gathered}
\langle x \mid 1\rangle=e^{-i k x}, \quad\langle x \mid 2\rangle=e^{i k x}, \\
\left|1_{s}\right\rangle=\chi^{(\mu)}(\pi), \quad\left|2_{s}\right\rangle=\chi^{(\mu)}(\pi),
\end{gathered}
$$

and similarly in lead II

$$
\begin{gathered}
\left\langle x^{\prime} \mid 1^{\prime}\right\rangle=e^{-i k x^{\prime}}, \quad\left\langle x^{\prime} \mid 2^{\prime}\right\rangle=e^{i k x^{\prime}}, \\
\left|1_{s}^{\prime}\right\rangle=\chi^{(\mu)}(0), \quad\left|2_{s}^{\prime}\right\rangle=\chi^{(\mu)}(0) .
\end{gathered}
$$

Note that the subscript $s$ refers to the spin degree of freedom. Since $x^{\prime}=x+2 a$, where $x^{\prime}$ is the local coordinate in lead II and $a$ is the ring radius, we have

$$
\left|1^{\prime}\right\rangle=e^{-i 2 k a}|1\rangle, \quad\left|2^{\prime}\right\rangle=e^{i 2 k a}|2\rangle .
$$

Hence the states of an electron in leads I and II are written as

$$
\begin{gathered}
\left|\Psi_{I}\right\rangle=\sum_{\mu, j=1,2} c_{j}^{\mu}|j\rangle \otimes\left|\mu_{s}\right\rangle, \\
\left|\Psi_{I I}\right\rangle=\sum_{\mu=1,2}\left(d_{1}^{\mu} e^{-i 2 k a}|1\rangle+d_{2}^{\mu} e^{i 2 k a}|2\rangle\right) \otimes\left|\mu_{s}^{\prime}\right\rangle .
\end{gathered}
$$

On the other hand it can be also verified that the spin states $\left(\chi(\pi) \equiv\left|\mu_{s}^{\prime}\right\rangle\right)$ in lead II are obtained from those in lead I $\left(\chi(0) \equiv\left|\mu_{s}\right\rangle\right)$ by the action of a unitary operator $\hat{U}$ $=\Sigma_{\mu, \nu} U^{\mu, \nu}\left|\mu_{s}\right\rangle\left\langle\nu_{s}\right|$, which acts only on the spin states

$$
\left|\mu_{s}^{\prime}\right\rangle=(-1)^{\mu+1} \hat{U}\left|\mu_{s}\right\rangle
$$

the corresponding matrix elements $U^{\mu, \nu}$ are given by

$$
\begin{gathered}
U^{1,1}=\cos \theta, \quad U^{1,2}=-\sin \theta, \\
U^{2,1}=\sin \theta, \quad U^{2,2}=\cos \theta .
\end{gathered}
$$

Hence the state $\left|\Psi_{I I}\right\rangle$ is written as

$$
\left|\Psi_{I I}\right\rangle=\sum_{\mu=1,2}\left(d_{1}^{\mu} e^{-i 2 k a}|1\rangle+d_{2}^{\mu} e^{i 2 k a}|2\rangle\right) \otimes\left(\hat{U}\left|\mu_{s}\right\rangle\right) .
$$

Consequently, the transfer matrix $\hat{F}$ which describes the spindependent scattering problem and connects the electron states $\left|\Psi_{I}\right\rangle$ and $\left|\Psi_{I I}\right\rangle$,

$$
\left|\Psi_{I I}\right\rangle=\hat{F}\left|\Psi_{I}\right\rangle,
$$

has the form

$$
\hat{F}=\sum_{\nu, \mu, i, j} U^{\nu, \mu} L_{j, i}^{\mu}|j\rangle\left\langle i|\otimes| \nu_{s}\right\rangle\left\langle\mu_{s}\right|,
$$

with the complex coefficients $L_{j, i}^{\mu}$ given by

$$
\begin{gathered}
L_{1,1}^{\mu}=e^{-i 2 k a}(-1)^{\mu+1} / t^{\mu}, \\
L_{1,2}^{\mu}=-e^{-i 2 k a}(-1)^{\mu+1} r^{\mu} / t^{\mu},
\end{gathered}
$$

$$
\begin{gathered}
L_{2,1}^{\mu}=e^{i 2 k a}(-1)^{\mu+1} r^{\mu} / t^{\mu}, \\
L_{2,2}^{\mu}=e^{i 2 k a}(-1)^{\mu+1}\left(t^{\mu}\right)^{2}-\left(r^{\mu}\right)^{2} / t^{\mu} .
\end{gathered}
$$

If the magnetic field is absent $\left(\Phi_{A B}=0\right)$, one can verify that $L_{i, j} \equiv L_{i, j}^{\mu}=L_{i, j}^{\mu}$ does not depend on the spin index and, by defining the operator $\hat{L}=\sum_{j, i} L_{j, i}|j\rangle\langle i|$ acting only on the spatial degree of freedom, the transfer matrix can be written as the tensor product

$$
\hat{F}=\hat{L} \otimes \hat{U} .
$$

In words, the spatial degree of freedom can be completely decoupled from that of the spin and be treated independently.

\section{Reflection and transmission matrices}

Assuming that there are only outgoing electrons to the right of the ring (region II) and the state of the incident electrons from the left is $|2\rangle \otimes\left|\chi_{s}^{i n}\right\rangle$ with arbitrary spin state $\left|\chi_{s}^{\text {in }}\right\rangle$, we can introduce the reflection operator $(\hat{R})$ and transmission $(\hat{T})$ operators, both of them acting only on the spin degree of freedom, as follows.

$$
\begin{gathered}
\left|\Psi_{I}\right\rangle=|2\rangle \otimes\left|\chi_{i n}\right\rangle+|1\rangle \otimes\left(\hat{R}\left|\chi_{s}^{i n}\right\rangle\right), \\
\left|\Psi_{I I}\right\rangle=|2\rangle \otimes\left(\hat{T}\left|\chi_{s}^{i n}\right\rangle\right) .
\end{gathered}
$$

Using Eq. (22) we find that the reflection and transition matrix elements satisfy the following equations:

$$
\begin{gathered}
F_{1,2}^{\nu, \mu}+\sum_{\gamma} F_{1,1}^{\nu, \gamma} R^{\gamma, \mu}=0, \\
F_{2,2}^{\nu, \mu}+\sum_{\gamma} F_{2,1}^{\nu, \gamma} R^{\gamma, \mu}=T^{\nu, \mu} .
\end{gathered}
$$

Consequently,

$$
\begin{gathered}
R^{\alpha, \beta}=-\frac{\sum_{\gamma}(-1)^{\alpha+\gamma} F_{1,1}^{\bar{\gamma}, \bar{\alpha}} F_{1,2}^{\gamma, \beta}}{F_{1,1}^{1,1} F_{1,1}^{2,2}-F_{1,1}^{1,2} F_{1,1}^{2,1}}, \\
T^{\alpha, \beta}=F_{2,2}^{\alpha, \beta}+\sum_{\gamma} F_{2,1}^{\alpha, \gamma} R^{\gamma, \beta},
\end{gathered}
$$

where we used the notations $\overline{1}=2$ and $\overline{2}=1$.

Using Eq. (24) and Eqs. (25a) and (25b) one can rewrite the reflection and transmission operators of Eqs. (29a) and (29b) in the form

$$
\begin{gathered}
\hat{R}=\sum_{\mu} r^{\mu}\left|\mu_{s}\right\rangle\left\langle\mu_{s}\right|, \\
\hat{T}=\hat{U} \sum_{\mu}(-1)^{\mu} t^{\mu}\left|\mu_{s}\right\rangle\left\langle\mu_{s}\right| .
\end{gathered}
$$

In the absence of the magnetic field we have $r \equiv r^{1}=r^{2}$ and $t \equiv t^{1}=-t^{2}$. Hence the operators $\hat{R}$ and $\hat{T}$ can be written as 


$$
\hat{R}=r \hat{I}, \quad \hat{T}=t \hat{U},
$$

where $\hat{I}=\Sigma_{\mu}\left|\mu_{s}\right\rangle\left\langle\mu_{s}\right|$ is the identity operator acting on the spin degree of freedom. In other words, the electron is reflected without changing its original spin polarization with the spin-independent probability amplitude $r$. On the other hand, the spins of those electrons which are transmitted with the spin-independent probability amplitude $t$ are rotated by the unitary operator $\hat{U}$.

\section{MULTIRING TRANSPORT}

For a chain of $N$ rings one can use the single-ring results in a straightforward manner if the rings touch each other. Associating the $i$ th ring with the transfer operator $\hat{F}(i)$ and using matrix multiplication, we can write the joint transfer operator, for the entire chain, as

$$
\hat{W}(1, \ldots, N)=\prod_{i=1}^{N} \hat{F}(i) .
$$

In component language, the joint transfer operator has the matrix elements

$$
\begin{aligned}
W_{j, i}^{\nu, \mu}(1, \ldots, N)= & \sum_{l=1}^{N-1} \sum_{\gamma_{l}, k_{l}} F_{j, k_{N-1}}^{\nu, \gamma_{N-1}}(N) F_{k_{N-1}, k_{N-2}}^{\gamma_{N-1}, \gamma_{N-2}}(N-1) \\
& \times \cdots F_{k_{2}, k_{1}}^{\gamma_{2}, \gamma_{1}}(2) F_{k_{1}, i}^{\gamma_{1}, \mu}(1) .
\end{aligned}
$$

The reflection and transmission matrix elements can be calculated as in the single-ring case, cf. Eqs. (28):

$$
\begin{gathered}
R^{\alpha, \beta}=-\frac{\sum_{\gamma}(-1)^{\alpha+\gamma} W_{1,1}^{\bar{\gamma}, \bar{\alpha}} W_{1,2}^{\gamma, \beta}}{W_{1,1}^{1,1} W_{1,1}^{2,2}-W_{1,1}^{1,2} W_{1,1}^{2,1}}, \\
T^{\alpha, \beta}=W_{2,2}^{\alpha, \beta}+\sum_{\gamma} W_{2,1}^{\alpha, \gamma} R^{\gamma, \beta} .
\end{gathered}
$$

Knowing the transition matrix one can calculate the conductance $G_{\mu}$ for electrons with spins polarized $(\mu=1,2)$ by

$$
G_{\mu}=\frac{e^{2}}{h} \sum_{\gamma=1,2}\left(T^{\gamma, \mu}\right)^{*} T^{\gamma, \mu} .
$$

Note that the subscripts 1 and 2 are associated to the upward and downward pointing spin polarization defined in Eq. (5). Then following Ref. 9 we can define the spin-up, $G_{1}$, and spin-down, $G_{2}$, conductances as follows:

$$
\begin{aligned}
& G_{1}=\frac{e^{2}}{h}\left[\left|T^{1,1}\right|^{2}+\left|T^{1,2}\right|^{2}\right], \\
& G_{2}=\frac{e^{2}}{h}\left[\left|T^{2,1}\right|^{2}+\left|T^{2,2}\right|^{2}\right] .
\end{aligned}
$$

If the incident electron spins are not polarized the conductance $G$ reads $G=G_{1}+G_{2}$, that is

$$
G=\frac{e^{2}}{h} \sum_{\gamma, \mu=1,2}\left(T^{\gamma, \mu}\right)^{*} T^{\gamma, \mu} .
$$
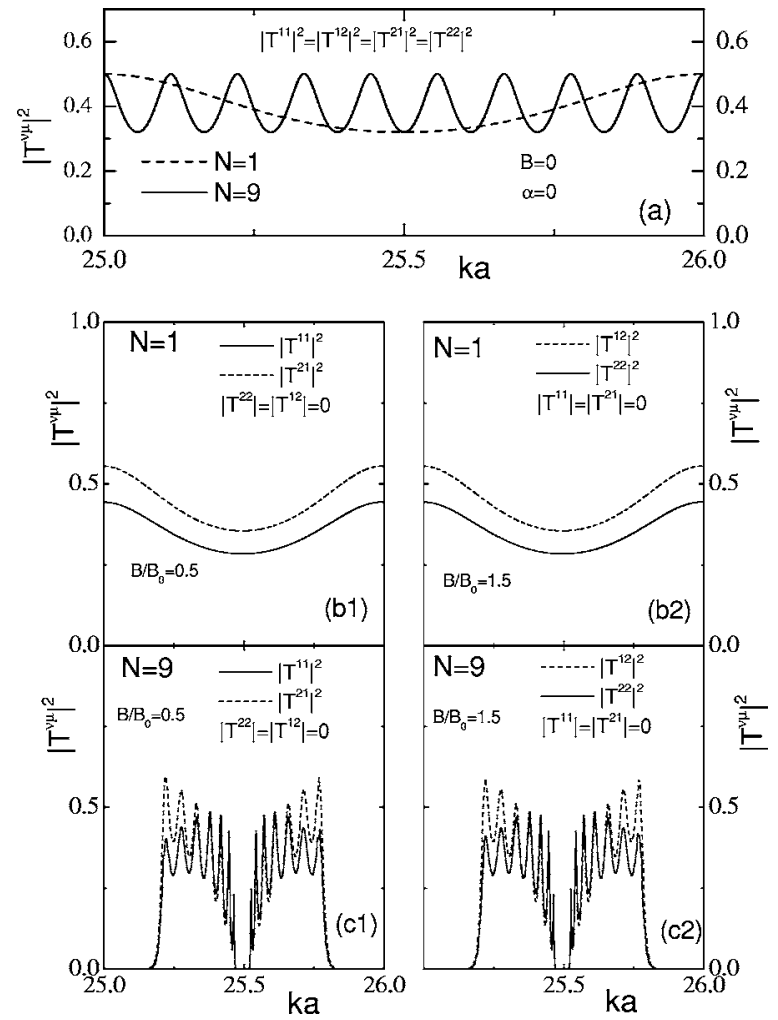

FIG. 2. (a) Transmission amplitudes $\left|T^{\mu, \nu}\right|^{2}$ vs $k a$ for zero SOI strength $(\alpha=0)$ and zero magnetic field $(B=0)$. The dashed curve is for a single ring and the solid curve for $N=9$ rings. (b1)-(c2): As in (a) for two finite values of $B$ and $\alpha=1.18 \alpha_{0}$.

The conductance at finite temperatures is given by

$$
G(T)=-\frac{e^{2}}{h} \int_{0}^{\infty} d E \frac{\partial f(E, T)}{\partial E} G(E, 0),
$$

where $f(E, T)$ is the Fermi function, $E$ the electron energy, $T$ the temperature, and $G(E, 0) \equiv G$ the conductance at zero temperature.

\section{NUMERICAL RESULTS}

Below we present numerical results for the magnetoconductance as a function of the SOI strength $\alpha$, the magnetic field $B$, and $k a$ where $k$ is the incident wave vector. We use the effective mass of InAs, $m^{*}=0.023 m_{0}$ and a ring radius $a=0.25 \mu \mathrm{m}$. Further, we measure $\alpha$ in units of $\alpha_{0}$ $=\hbar^{2} / 2 m^{*} a=0.6626 \times 10^{-11} \mathrm{eVm}$ and $B$ in units of $B_{0}$ $=\hbar / a^{2} e=10.53 \mathrm{mT}$. Note that $B_{0}$ is the magnetic field value which corresponds to the Aharonov-Bohm phase $\Phi_{A B}=\pi$. If not otherwise specified, we will set $G \equiv G(\alpha, B, k a)$. As for the Zeeman term, we neglected it from the outset because it is too small: for the above parameters and $k a=25$, we estimate $E_{F}=16.6 \mathrm{meV}$, SOI energy $\approx 1 \mathrm{meV}$, and Zeeman energy $\approx 0.043 \mathrm{meV}$ at $B=0.1 T$ while the highest $B$ value involved in the calculations is $B=4 B_{0} \approx 0.0432 \mathrm{mT}$.

In Fig. 2(a) we show the transmission amplitudes $\left|T^{\mu, \nu}\right|^{2}$ vs $k a$, in the absence of SOI $(\alpha=0)$ and of the magnetic field $(B=0)$, through a single ring (dashed curve) and through a 


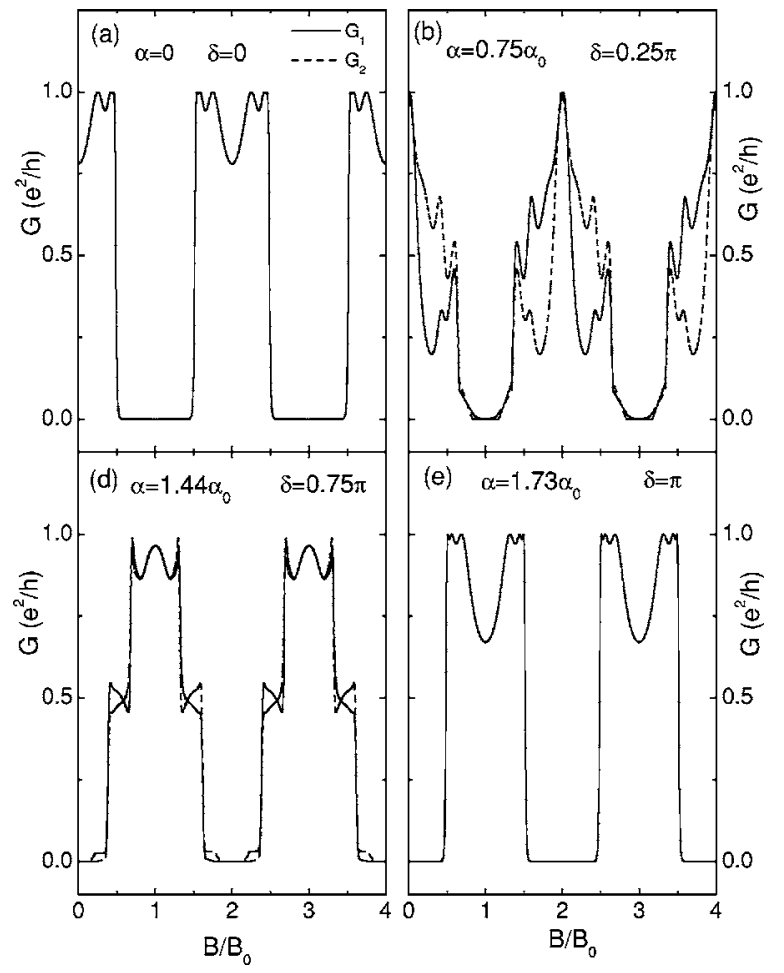

FIG. 3. Magnetoconductance $G$ vs field $B$ through a chain of $N=9$ rings for $k a=25.25$ and various values of $\alpha$. The solid and dashed curves are for the spin-up $\left(G_{1}\right)$ and spin-down $\left(G_{2}\right)$ conductances.

chain of $N=9$ identical rings (solid curve) within one period of $k a$. As expected, the number of oscillations is equal to the number of rings. In panels (b1)-(c2) we show again the transmission amplitudes for two different values of the magnetic field. Upon contrasting the many-ring case with the one for $B=0$, we see how well-defined bands and gaps develop for finite $B$. Upon further increasing $N$, a nearly square-wave magnetoconductance profile results. Further, in panels (b1),(b2),(c1), and (c2) the value of $\alpha=1.18 \alpha_{0}$ was chosen so that the corresponding Aharonov-Casher phase $\Phi_{A C}^{(1)} \equiv \delta$ $=\pi / 2$. Since $\Phi_{A C}^{(1)}+\Phi_{A C}^{(2)}=-2 \pi$ we have $\Phi_{A C}^{(2)}=-5 \pi / 2$. On the other hand, in panels (b1) and (c1) the magnetic field is $B$ $=0.5 B_{0}$ and gives $\Phi_{A B}=\pi / 2$; hence the combined AharanovBohm-Casher phase $\Phi_{A B C}^{(1)}$ has the value $\Phi_{A B C}^{(1)}=\Phi_{A C}^{(1)}+\Phi_{A B}$ $=\pi$ for $\mu=1$ and $\Phi_{A B C}^{(2)}=\Phi_{A C}^{(2)}+\Phi_{A B}=-2 \pi$ for $\mu=2$. This renders the ring or the chain totally opaque for electrons with spin polarization $\mu=1$ and transparent for $\mu=2$, cf. Eq. (15). In the right panels (b2) and (c2) the magnetic field is $B$ $=1.5 B_{0}$. This gives a "constructive" phase $\Phi_{A B C}^{(1)}=2 \pi$ for $\mu$ $=1$ and a "destructive" one $\Phi_{A B C}^{(2)}=-3 \pi / 2$ for $\mu=2$. As indicated in Fig. 2, in both cases some $T^{\mu \nu}$ matrix elements vanish for the first ring and thus for the chain; this follows directly from Eqs. (15a) and (15b).

In Fig. 3 we show $G$ vs $B$ for several values of $\alpha, N=9$, and $k a=25.25$. The dashed and solid curves represent the spin-up $G_{1}$ and spin-down $G_{2}$ conductances, respectively. Notice the wide ranges of the field $B$, for some values of $\alpha$, in which the conductance vanishes. This results from the interference between spins traveling clockwise and counter-

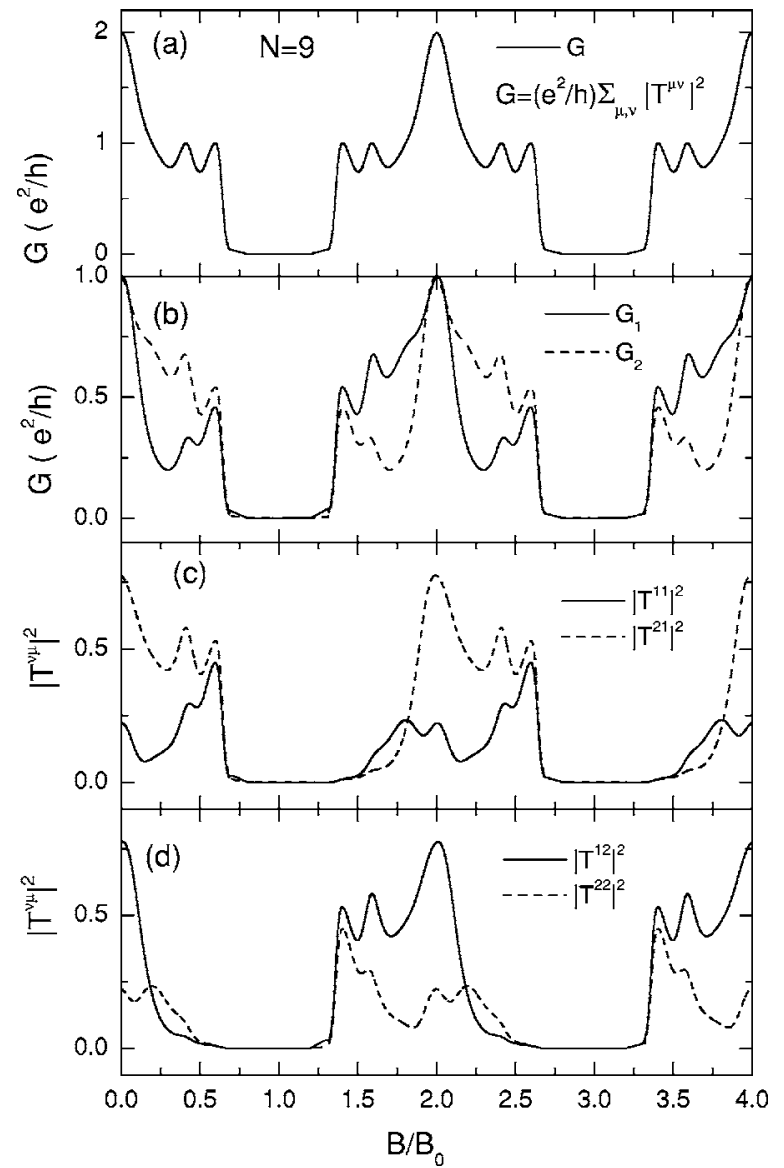

FIG. 4. (a) Magnetoconductance $G$ vs field $B$ through a chain of $N=9$ rings for $\alpha=0.75 \alpha_{0}$ and $k a=25.25$. (b) Spin-up $G_{1}$ and spindown $G_{2}$ conductances vs $B$. (c) and (d) Transmission amplitudes $\left|T^{\mu, \nu}\right|^{2}$ vs $B$. Their sum gives the magnetoconductance $G$ shown in (a).

clockwise being destructive as the corresponding AharonovBohm-Casher phases $\Phi_{A B C}^{(\mu)}$ are equal to a multiple of $\pi$; in this case we have $G_{1}=G_{2}$. To further stress this point, in Fig. 4 we show $G$, in panel (a), the spin-up $G_{1}$ and spin-down $G_{2}$ in panel (b), and the various transmission amplitudes $\left|T^{\mu, \nu}\right|^{2}$ in panels (c) and (d), all vs $B$ for $\alpha=0.75 \alpha_{0}$. Notice that the gaps in $G, G_{1}$, and $G_{2}$ occur at the same values of $B$. Also, if the incident carriers are up (down) polarized, the conductance is $G_{1}\left(G_{2}\right)$. As for the apparent symmetry between certain transmission amplitudes $T^{\mu \nu}$ between panels (c) and (d), it can be understood with the help of Eqs. (29a), (15), and (15b); it will be detailed elsewhere.

In Figs. 5(a)-5(d), we show the grayscale $\left(k a, B / B_{0}\right)$ contour plots of the magnetoconductance $G$ vs $B$ for various values of $\alpha, N=9$ rings, and $k a=25.25$. The values of $\delta$ are those of the corresponding Aharonov-Casher phase. The $(B, \alpha)$ profile of the chain is shown in (e) and the gray color meter in (f). We notice the existence of wide, dark regions in which $G$ vanishes. The near periodic character of $G$ upon varying $k a$ can be understood with reference to Eq. (15) in which we have $q=\left[k^{2}+\left(\omega_{s o} / 2 a \Omega\right)^{2}\right]^{1 / 2}$. For the value of $k a$ used we have $k a \gg \omega_{s o} / 2 \Omega$ and the periodicity of $G$ is evident. With respect to the $B$ dependence and again with ref- 

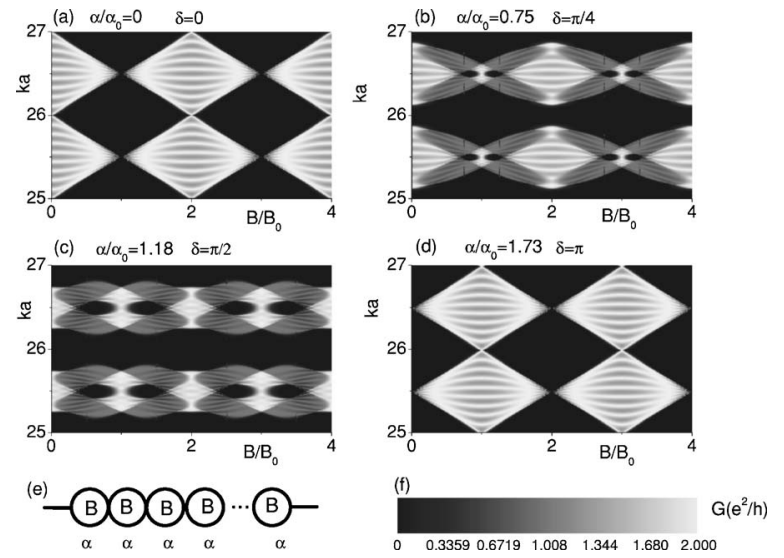

FIG. 5. (a)-(d) $\left(k a, B / B_{0}\right)$ contour plots of the magnetoconductance $G$ through a chain of $N=9$ rings for various values of $\alpha$. (e) $(B, \alpha)$ profile of the chain. (f) Gray color meter.

erence to Eq. (15), we see how the $\delta=\pi$ pattern is shifted with respect to that for $\delta=0$.

Similar contour plots of the magnetoconductance $G$, but now with $\alpha$ on the $x$ axis, are shown in Fig. 6 for various values of $B$. The other parameters are the same as in Fig. 5. Different from Fig. 5, here we see that upon increasing $\alpha$ the contour plots are less symmetric than those of Fig. 5 since the inequality $k a \gg \omega_{s o} / 2 \Omega$ is progressively violated.

We now present contour plots in which $\alpha$ and/or $B$ are varied periodically from ring to ring. In Fig. 7 we show the contour plots similar to those of Fig. 5 but with $\alpha$ modulated as shown in panel (e). One notices the similarity of the contours in both figures but also some differences, e.g., the $\delta$ $=0$ results are identical; there are wider dark areas in Fig. 7 , and there is no simple shift between the $\delta=0$ and the $\delta=\pi$ panels. The results shown in Fig. 7 are similar to those of Ref. 10 for a single waveguide with a periodically varied $\alpha$ at $B=0$.

Other contour plots with periodic modulation of $\alpha$ and $B$ are shown in Fig. 8. We notice immediately that (i) for $\delta$ $=0$ the periodicity along $k a$ is doubled, (ii) the dark areas are quite wider than in the previous figures, and (iii) the absence
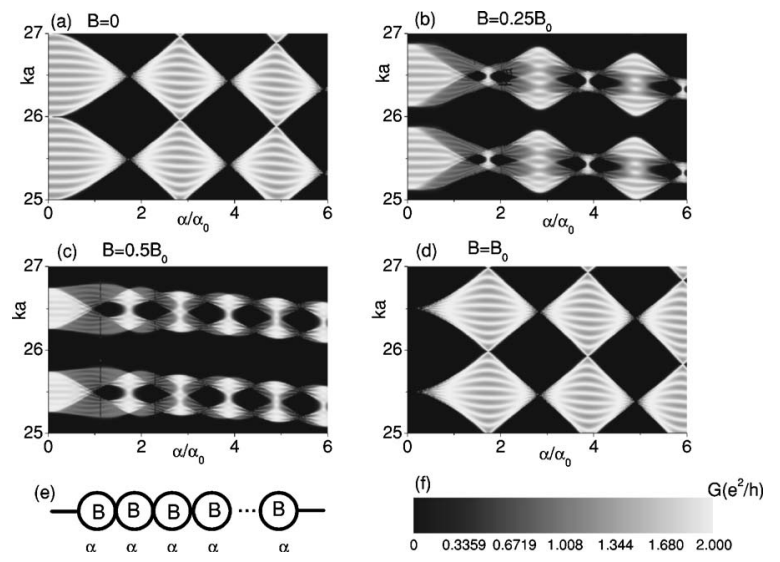

FIG. 6. (a)-(d), $\left(k a, \alpha / \alpha_{0}\right)$ contour plots of the magnetoconductance $G$ through a chain of $N=9$ rings for various values of $B$. (e) and (f) Same as in Fig. 5.
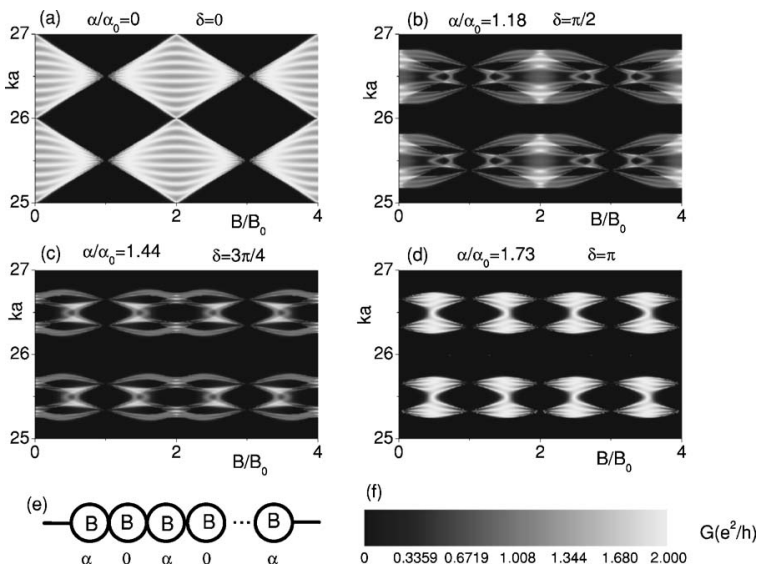

FIG. 7. (a)-(d), (f) Same as in Fig. 5 but now for a chain with $\alpha$ modulated. (e) $(B, \alpha)$ profile of the chain with uniform $B$ and $\alpha$ varied periodically from ring to ring.

of any transmission for $\delta=\pi$ where the first ring is totally reflective.

The various gaps, dark areas in Figs. 5-8 in which $G$ vanishes can be understood with the help of Eqs. (2) and (15). In Figs. 5 and 6 this stems directly from the periodicity of the transmission and reflection coefficients with respect to the phases $\Phi_{A B}$ and $\Phi_{A C}$. As for Figs. 7 and 8 upon periodically varying the strength $\alpha$ and/or the field $B, \omega_{s o}$ and the energy levels change from ring to ring thus creating the usual superlattice barriers or wells. Depending then on the incident electron's energy one has the usual gaps or bands in the transmission.

The influence of the temperature on $G$ is shown in Fig. 9 . In all panels the solid and dashed curves show the spin-up $\left(G_{1}\right)$ and spin-down $\left(G_{2}\right)$ conductances, respectively, and $k a=25.25$. The values $\alpha=0.75 \alpha_{0}, \quad \alpha=1.18 \alpha_{0}$, and $\alpha$ $=1.73 \alpha_{0}$ correspond to a Aharonov-Casher phase equal to $\pi$, $\pi / 2$, and $\pi / 4$, respectively. Contrasting the upper panels, for temperature $T=0.19 \mathrm{~K}$, with the lower ones, for temperature $T=1.96 \mathrm{~K}$, we see that the main effect of increasing the temperature is to round off the structure seen at the lower temperature and the resulting disappearance of some fine struc-
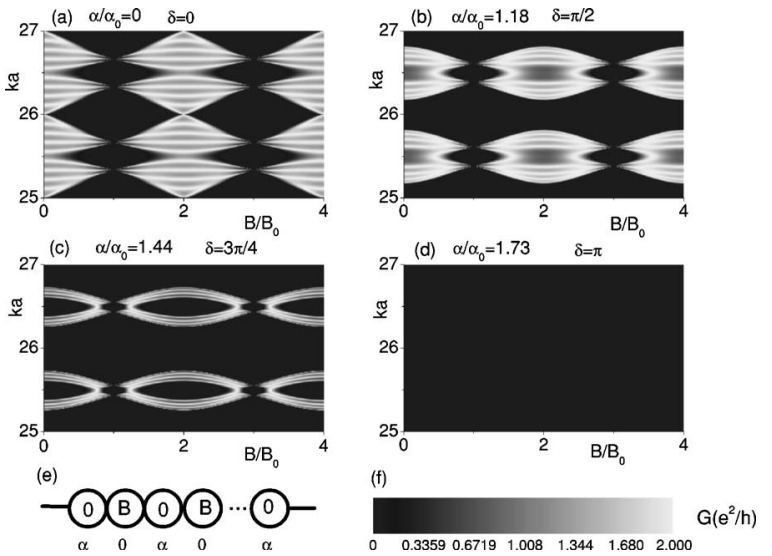

FIG. 8. (a)-(d), (f) Same as in Fig. 5 but now with $\alpha$ and $B$ modulated. (e) $(B, \alpha)$ profile of the chain with $\alpha$ and $B$ varied periodically from ring to ring. 

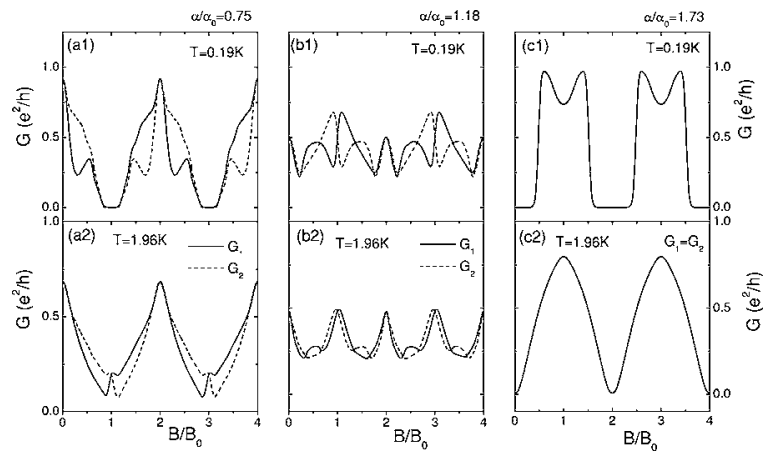

FIG. 9. Magnetoconductance $G$ vs field $B$ for two temperatures, $T=1.96$ and $0.196 \mathrm{~K}$, and three different values of $\alpha$ corresponding to a Aharonov-Casher phase equal, from left to right, to $\pi / 4, \pi / 2$, and $\pi$. In all panels the solid (dashed) curves show the spin-up $G_{1}$ (spin-down $G_{2}$ ) conductance. On the scale used the results for $T$ $=0.6 \mathrm{~K}$ are indistinguishable from those for $T=0.196 \mathrm{~K}$ in the upper panels.

ture. At the same time we see how $G_{1}$ and $G_{2}$ are affected by the changes in $\alpha$ or the Aharonov-Casher phase. We further notice that for a Aharonov-Casher phase equal to $\pi$, the up $\left(G_{1}\right)$ and down $\left(G_{2}\right)$ conductances are equal, cf. Eq. (15).

\section{CONCLUDING REMARKS}

We studied ballistic magnetotransport through chains of circular one-dimensional rings in the presence of SOI. The evaluated magnetoconductance shows clearly a periodic dependence on the incident electron's energy, through the parameter $k a$, the magnetic field $B$, and the strength $\alpha$ of the SOI. Depending on the values of these parameters and of the Aharonov-Casher phase $\delta$, a variety of periodic and nearly square-wave patterns results, cf. Figs. 5 and 6. Interestingly, as Fig. 4 demonstrates, the gaps in the conductance occur whether the incident electrons are spin polarized or not. Ac- cordingly, the results could be relevant to the development of a spin-transistor for which a spin-dependent and as binary as possible conductance output is necessary.

In addition, we studied the influence of periodic modulations of $\alpha$ and $B$ on the magnetoconductance through a chain of rings. As in the $B=0$ case, ${ }^{4}$ we have again various periodic conductance patterns that are similar to those involving uniform $B$ and $\alpha$ but with a somewhat stronger square-wave character, cf. Figs. 7 and 8 . In all cases, the periodicity of the pattern stems from that of the transmission and reflection coefficients of a single ring and/or that of the externally imposed $B$ and/or $\alpha$ profile which entails the same modification, from ring to ring, of the energy levels given by Eq. (2).

As for the temperature dependence of the magnetoconductance, the results are similar to those for a single ring: ${ }^{3}$ the main effect of raising the temperature is to round off the patterns seen at zero temperature.

The results presented here are valid for chains of strictly one-dimensional rings. They can be extended to rings of finite width $w$ provided the inequality $w \ll a$ holds and, e.g., a square-well confinement is assumed along the radial direction. In this case the radial and angular motion are decoupled and the energy levels, given by Eq. (2), are shifted by $\hbar^{2} l^{2} / 2 m^{*} w^{2}$, where $l$ is an integer. The results presented above correspond to the lowest $l=1$ mode. If mode mixing is considered, a further study is necessary. However, on account of similar studies in straight waveguides, ${ }^{11}$ we expect more complicated but qualitatively the same patterns.

\section{ACKNOWLEDGMENTS}

This work was supported by the Belgian Science Policy, the Flemish Concerted Action Programme (GOA), the Flemish Science Foundation (FWO-Vl), the EU-CERION programme, the Flemish-Hungarian Bilateral Programme, and by the Canadian NSERC Grant No. OGP0121756. One of us (B. M.) is supported by DWTC.
${ }^{1}$ E. I. Rashba, Sov. Phys. Solid State 2, 1109 (1960).

${ }^{2}$ Y. Aharonov and A. Casher, Phys. Rev. Lett. 53, 319 (1984); A. G. Aronov and Y. B. Lyanda-Geller, ibid. 70, 343 (1993); S. L. Zhu and Z. D. Wang, ibid. 85, 1076 (2000); D. Frustaglia, M. Hentschel, and K. Richter, ibid. 87, 256602 (2001); J. B. Yau, E. P. De Poortere, and M. Shayegan, ibid. 88, 146801 (2003); D. Bercioux, M. Governale, V. Cataudella, and V. M. Ramaglia, ibid. 93, 056802 (2004); Y. S. Yi, T. Z. Qian, and Z. B. Su, Phys. Rev. B 55, 10631 (1997); D. Frustaglia and K. Richter, ibid. 69, 235310 (2004).

${ }^{3}$ B. Molnár, F. M. Peeters, and P. Vasilopoulos, Phys. Rev. B 69, 155335 (2004).
${ }^{4}$ B. Molnár, P. Vasilopoulos, and F. M. Peeters, Appl. Phys. Lett. 85, 612 (2004).

${ }^{5}$ F. E. Meijer, A. F. Morpurgo, and T. M. Klapwijk, Phys. Rev. B 66, 033107 (2002).

${ }^{6}$ D. Grundler, Phys. Rev. Lett. 84, 6074 (2000).

${ }^{7}$ S. Griffith, Trans. Faraday Soc. 49, 345 (1953).

${ }^{8}$ J. B. Xia, Phys. Rev. B 45, 3593 (1992); T. Choi, S. Y. Cho, C. M. Ryu, and C. K. Kim, ibid. 56, 4825 (1997).

${ }^{9}$ F. Mireles and G. Kirczenow, Phys. Rev. B 64, 024426 (2001).

${ }^{10}$ X. F. Wang and P. Vasilopoulos, Appl. Phys. Lett. 83, 940 (2003).

${ }^{11}$ X. F. Wang and P. Vasilopoulos, Phys. Rev. B 68, 035305 (2003). 